\title{
CHEAT-PROOF COOPERATION STRATEGIES FOR WIRELESS LIVE STREAMING SOCIAL NETWORKS
}

\author{
W. Sabrina Lin*, H. Vicky Zhao ${ }^{\dagger}$ and K. J. Ray Liu* \\ * ECE Dept., University of Maryland, College Park, MD 20742 USA \\ ${ }^{\dagger}$ ECE Dept., University of Alberta, Edmonton, AB T6G 2V4 Canada
}

\begin{abstract}
Multimedia social network analysis is an emerging research area, which analyzes the behavior of users who share multimedia content and investigates the impact of human dynamics on multimedia systems. Users watching live streaming in the same wireless network share the same backbone connection to the Internet, thus they might want to cooperate with each other to obtain better video quality. These users form a wireless live-streaming social network and every user wishes to watch video with as high as possible quality while paying as less as cost for cooperation. Thus full cooperation cannot be guaranteed and the cooperation strategy must give incentives to the users. This paper proposes a game-theoretic framework to model user behavior and designs incentive-based strategies to stimulate user cooperation in wireless live streaming. We analyze the Pareto optimality and time-restricted bargaining equilibrium of the game. We also take into consideration selfish users' cheating behavior and propose cheat-proof strategies. Both our analytical and simulation results show that the proposed strategies can effectively stimulate user cooperation, achieve cheat free and help provide reliable services.
\end{abstract}

Index Terms - Multimedia social network, game theory, behavior dynamics, wireless live streaming

\section{INTRODUCTION}

With the explosive advance communication technologies and multimedia signal processing, over millions of users share multimedia data over Internet. These users interact with each other and form large-scale multimedia social networks. Peer-to-peer live streaming network [1] is one of the biggest multimedia social networks on the internet, and recent development on wireless local area network (WLAN) enable users to utilize WLAN with low cost and high quality of service.Live-streaming users in the same WLAN form a wireless live-streaming social network, and due to the instability of wireless channel, the cooperation among users are even more important than live-streaming networks in wired network.

Users in the wireless live-streaming social networks may have different types, including laptops, PDAs, cellphones, and mp3/video players. Different types of social network members have different demand of quality and power, for instance, the laptop users would prefer higher resolution of videos and are willing to use more transmission power for cooperation than PDA users. They are likely to manipulate any incentive system to maximize their own payoff. Game theory [2] is a proper tool to model the interaction among peers, and to analyze the optimal and cheat-proof cooperation strategies. In this paper, we focus on providing incentives for two members in the social network to cooperate with each other. In the orig-

The authors can be reached at wylin@eng.umd.edu, vzhao@ece.ualberta.ca, and kjrliu@eng.umd.edu. inal system, all the members directly download the video chunks from the server. However, all the users share the same link through the access point to the Internet and each user has different playback time and ask for different chunks at the same time. Also there are other users in the wireless network accessing Internet simultaneously. Thus the link might be busy and some chunks can not be received by the end users in time for the playback time. And many of the users in the wireless networks have high mobility, with which they would change physical positions from time to time and the quality of network connections is also unstable. These reasons would stimulate users in the wireless live-streaming social networks to cooperate with each other.

In the literature,[3], propose an auction-based mechanism for wireless peer-to-peer (P2P) file sharing, and [4] studied the capacity of user-cooperation in wireless network. A game theoretic framework was proposed in [5] for P2P file sharing and [6], [7] model to provide incentives for cooperation in $\mathrm{P} 2 \mathrm{P}$ file sharing. The works in [8], [9] study the cooperation mechanism for P2P live streaming networks. However, there is no prior work on user cooperation, cheating behavior of selfish users and do not address the cheat-proof issues in wireless live-streaming social networks.

In this paper, we will focus on designing cooperation stimulation strategies for wireless live streaming social networks using a game theoretic framework. We first model the cooperation between two users as a Bayesian game and investigate the Bayseian-Nash equilibria. Since this game usually has multiple equilibria, we then investigate how to apply extra optimality criteria, such as Pareto optimality, bargaining, and cheat-proofing, to further refine the obtained equilibrium solutions. Such analysis aims to stimulate each pair of user in the wireless live-streaming game to cooperate with each other and achieve better performance.

The rest of this paper is organized as follows. Section 2 introduces the wireless live-streaming system model. Section 3 studies the two-player game and the equilibria. In Section 4 we show simulation results to evaluate the performance of the proposed strategies. Finally, Section 5 concludes this paper.

\section{SYSTEM MODEL}

In this section, we first describe the model of wireless live streaming system and how two users in a wireless live streaming social network cooperate with each other. We then define the payoff function and introduce the game-theoretic framework of the user dynamics.

\subsection{Wireless Live Streaming Model}

Figure 1 shows the architecture of a wireless video live-streaming social network. The wireless network service is provided by an access point which is connected to the Internet. The video bit stream is divided into media chunks of $M^{\prime}$ bits in the original server, and are channel-coded to $M$ bits, equivalent to $t$ seconds piece by the ac- 


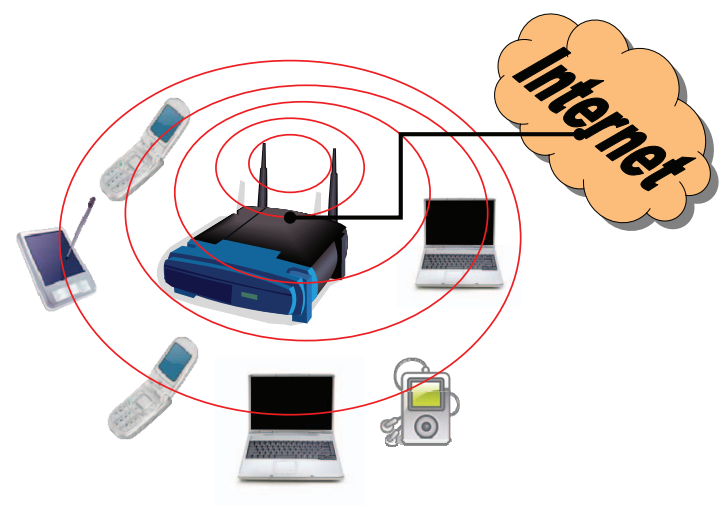

Fig. 1. Illustration of a wireless live-streaming social network

cess point depending on the communication protocol. All the chunks are available at an original server in the Internet. Here we assume a channel with bandwidth $B$ and additive white Gaussian noise with variance $\sigma_{n}^{2}$ that is available for members in the social network to communicate with each other.

We assume that two users, $u_{1}$ and $u_{2}$ in the social network try to cooperate with each other by exchanging chunks. Each user has a buffer of length $L$, which keeps $L_{f}$ chunks to be played, and $L-$ $L_{f}$ chunks that have been played for cooperation. First $u_{1}$ and $u_{2}$ exchange the information about the availability of each chunk in the other's buffer, and the transmission power $P_{1}$ and $P_{2}$ that $u_{1}$ and $u_{2}$ use to transmit the chunks, respectively. To ensure the quality of cooperation, intuitively the users will not cooperate with people who use too less power for cooperation. Hence we assume $P_{1}$ and $P_{2}$ are larger than the minimum transmit power $P_{\min }$. The chunk exchange is done on a round by round basis. At the beginning of each round, each user sends requests to the other users, and at the same time keep downloading from the original server. Each user are allowed to send multiple requests in each round and also answer multiple requests. Let $\tau$ be the duration of each round.

\subsection{Two-Player Game Model}

To simplify the analysis, we start from modeling the cooperation in each round be a two-person game with single-layer video coding structure. In this scenario, every chunk has the same value, thus the users will always request chunks closest to playback time that they need. Assume in the original structure, every user in the wireless live-streaming social network only asks the original server in the Internet for the media chunks, and two of them, $u_{1}$ and $u_{2}$, want to see if they can cooperate with each other for to get a better-quality video. We model the interactions between $u_{1}$ and $u_{2}$ as the following game:

- Players and player types: There are two players, $u_{1}$ and $u_{2}$, in this game. Each player $u_{i}$ has a type $\theta_{i} \in\{$ laptop, PDA, cellphone $\}$. Users with different type will have different cost of sharing chunks and gain of obtaining chunks.

- Strategies: In each round, the two players first exchange their buffer information, and then send the chunk requests to each other. Upon receiving the chunk requests, each player $u_{i}$ decides how many chunks he/she will send to the other user in this round. We define the number of chunks $u_{i}$ agrees to send as his/her strategy $a_{i} \in \mathbb{Z}$. Note that the two users are using the same channel, so the bits to be transmitted within a round can not be larger than the channel capacity, which equals to bandwidth times the logarithm of $1+$ signal to noise ratio. Therefore, the constraint of strategy profile $\left(a_{1}, a_{2}\right)$ is

$$
\frac{a_{1}}{\log \left(1+P_{1} / \sigma_{n}^{2}\right)}+\frac{a_{2}}{\log \left(1+P_{2} / \sigma_{n}^{2}\right)} \leq \frac{\tau B}{M} .
$$

If (1) is not satisfied and the collision happens, neither of the user will receive any chunks successfully.

- Utility function: The utility function $\pi_{i}$ of $u_{i}$ is considered as the gain of receiving chunks (with respect to the opponent's action) minus the cost of sending chunks (his/her own action). Since the members in the wireless live-streaming social network are using mobile devices, the battery energy is the most limited resource. Hence the cost of cooperation is considered as the energy for transmission where each type of player would give a different weight to the cost of its energy. For example, clients running on tight energy budget bear a higher cost than those with powerful batteries. Let $c_{i}$ be the cost per unit energy for $u_{i}$, and $g_{i}$ be $u_{i}$ 's gain of completely received one chunk. Then the players' payoffs for each round are formulated as follows, provided that the strategy profile $\left(a_{1}, a_{2}\right)$ being taken:

$$
\begin{aligned}
& \pi_{1}\left(a_{1}, a_{2}\right)=a_{2} g_{1}-a_{1} c_{1} \frac{M P_{1}}{B \log \left(1+\frac{P_{1}}{\sigma_{n}^{2}}\right)} \\
& \pi_{2}\left(a_{1}, a_{2}\right)=a_{1} g_{2}-a_{2} c_{2} \frac{M P_{2}}{B \log \left(1+\frac{P_{2}}{\sigma_{n}^{2}}\right)} .
\end{aligned}
$$

Let $\pi\left(a_{1}, a_{2}\right)=\left(\pi_{1}\left(a_{1}, a_{2}\right), \pi_{2}\left(a_{1}, a_{2}\right)\right)$ be the payoff profile, $M P_{1} / B \log \left(1+P_{1} / \sigma_{n}^{2}\right)$ be $K_{1}$, and $M P_{2} / B \log \left(1+P_{2} / \sigma_{n}^{2}\right)$ be $K_{2}$. It is reasonable to assume that $g_{i} \geq c_{i} K_{i}$ and there exists a $C_{\max }$ with $c_{i} K_{i} \leq C_{\max }$. Here $c_{i}$ and $g_{i}$ are the private information of the users depends on the user's type and are not known to others. We assume the users do not exchange their private information, i.e., their types, then this is a game with incomplete information. But the users have the belief of the probability of the other users' type, which is independent of their own type. Let $p_{1}, p_{2}, p_{3}$ be the probability of a user being a laptop, PDA, and cellphone, respectively.

\section{OPTIMAL STRATEGIES ANALYSIS}

\subsection{Repeated Game Model}

It is easy to check that, if the above game will only be played for one time, the only Bayesian-Nash equilibrium is $(0,0)$, which means no one will answer the other's requests. According to the backward induction principle [10], there will also be no cooperation between the two users when the repeated game will be played for finite times with game termination time known to both players. Therefore, in both circumstances, the only optimal strategy for both players is to always play noncooperatively.

However, in live streaming scenario, these two players will interact many rounds and no one can know exactly when the other player will quit the game. Thus we can model the dynamics between $u_{1}$ and $u_{2}$ as an infinitely repeated game, and we will show that cooperative strategies can be obtained in this realistic model. Let $s_{i}$ denote player $i$ 's behavior strategy, and let $\mathbf{s}_{1}, \mathbf{s}_{2}$ denote the strategy profile. Next, we consider the following utility function of the infinitely repeated game:

$$
U_{i}(\mathbf{s})=\lim _{T \rightarrow \infty} \sum_{t=0}^{T} u_{i}(\mathbf{s})
$$

Now, we analyze Bayesian-Nash equilibriums for the infinitely repeated game with utility function $U_{i}$. According to Folk theorem [10], there exists at least one Bayesian-Nash equilibrium to achieve 


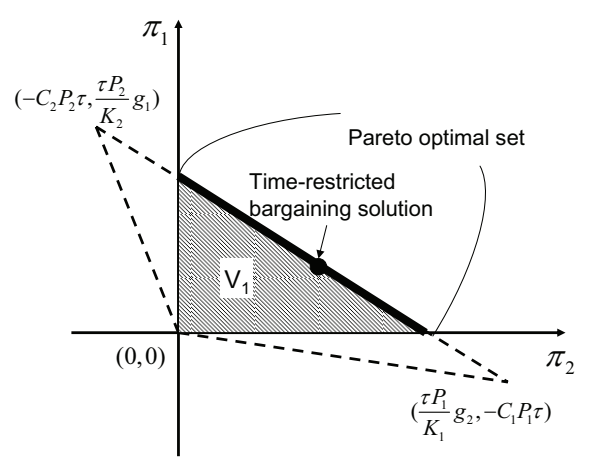

Fig. 2. Feasible and Enforceable payoff profiles

every feasible and enforceable payoff profile, where the set of feasible payoff profiles for the above game is:

$$
\begin{gathered}
V_{0}=\text { convex hull }\left\{v \mid \exists\left(a_{1}, a_{2}\right) \text { with } \pi\left(a_{1}, a_{2}\right)=v\right\} \\
\text { where } a_{1}, a_{2} \text { satisfy }(1)
\end{gathered}
$$

and the set of enforceable payoff, denoted by $V_{1}$, can be easily derived:

$$
V_{1}=\left\{v \mid v \in V_{0} \quad \text { and } \quad v \geq(0,0)\right\}
$$

Figure 2 illustrates the both feasible region and the enforceable region: the feasible region is inside the triangle bounded by dashed lines, and the enforceable feasible set $V_{1}$ is the shaded region shown in Figure 2. It is clear that there exists an infinite number of Bayesian-Nash equilibriums (BNE). To simplify our equations, in this paper, we use $\mathbf{x}=\left(x_{1}, x_{2}\right)$ to denote the set of BNE strategies corresponding to the enforceable payoff profile $\left(x_{2} g_{1}-\right.$ $\left.x_{1} c_{1} K_{1}, x_{1} g_{2}-x_{2} c_{2} K_{2}\right)$.

From the above analysis, one can see that the infinitely repeated game has infinite number of equilibriums, and apparently, not all of them are simultaneously acceptable. For example, the payoff profile $(0,0)$ is not acceptable from both players' point of view. Therefore, in this section, we will discuss how to refine the equilibriums based on new optimality criteria to eliminate those less rational and which equilibrium is cheat-proof.

\subsection{Nash Equilibrium Refinement}

The following optimality criteria will be considered in this section: Pareto optimality, proportional fairness, and absolute fairness.

Pareto Optimality: A payoff profile $v \in V_{0}$ is Pareto Optimal if and only if there is no $v^{\prime} \in V_{0}$ that $v_{i}^{\prime} \geq v_{i}$ for all $i \in N$ [2]. Pareto Optimality means no one can increase his/her payoff without degrade other's, which the rational players will always go to.

It's clear that from Figure 2 that the solid segment between $\left(-C_{2} P_{2} \tau\right.$ $\left.g_{1} \tau P_{2} / K_{2}\right)$ and $\left(g_{2} \tau P_{1} / K_{1},-C_{1} P_{1} \tau\right)$ in the first quadrant is the Pareto Optimal set.

Time-restricted bargaining solution: Since the players' action $\left(a_{1}, a_{2}\right)$ has to satisfy 1 , and both players are rational, which means they will try to maximize the quality of their live streaming by asking as many chunks as possible in each round. Every user will request all the chunks that his/her opponent has and he/she needs. Thus the users have to bargain for their chunk-request quota for every round to ensure the total number of bits to be transmitted is not larger than the channel capacity. Also, the gain of receiving a chunk is timesensitive. For instance, if users cannot reach an agreement on time and the chunk does not arrive before the playback time, then a user has no gain by receiving that chunk.

We model the time-restricted process for round $k$ as follows: one user offers an action pair $\left(a_{1}^{(1)}, a_{2}^{(1)}\right)$ first, and the other user can decide whether to accept this offer or to reject and offer back a action pair $\left(a_{1}^{(2)}, a_{2}^{(2)}\right)$. This process continues until both players agree on the offer. If the users reach agreement at the $j^{\text {th }}$ action pair, then $g_{i}$ decreases to $\delta_{i}^{j-1}\left(L C_{k, i}\right) g_{i}$ for $i=1$ or 2 , where $\delta_{i}\left(L C_{k, i}\right)$ is the discount factor for $u_{i}, L C_{k, i}=\left\{I_{1}, \ldots, I_{q}\right\}$ denotes the indexes of chunks $u_{i}$ wants to ask in the $k^{t h}$ round, and $I(k)$ denotes the chunk playing at the beginning of $k^{\text {th }}$ round. Suppose the first $q^{\prime}$ terms in $L C_{k, i}$ are smaller than $I(k)+\tau / t$, we define the discount factor as follows:

$$
\delta_{i}\left(L C_{k, i}\right)=1-\frac{\sum_{i=1}^{q^{\prime}} \frac{\tau}{t}-\left(I_{i}-I(k)\right)+\left(q-q^{\prime}\right) * d}{\frac{\tau}{t}\left(\frac{\tau}{t}+1\right) / 2+\left(L-L_{f}\right) * d},
$$

where $d_{i} 1$ is the discount constant of the chunks that will be played after the $k+1^{\text {th }}$ round begins.

In the time-restricted bargaining model, the user who makes the first offer is in advantage. Therefore, a reasonable model for the livestreaming game is the two users take turns to take the first move.

Since both players' payoffs decrease as the time for bargaining increases, the first mover would seek the equilibrium and offer at the first bargaining round for his/her maximum payoff. Let $\delta_{1}$ and $\delta_{2}$ are the averaged discount factor over for $u_{1}$ and $u_{2}$ over all rounds. The Pareto-optimal equilibrium pair $\left(\left(x_{1}^{(1)}, x_{2}^{(1)}\right),\left(x_{1}^{(2)}, x_{2}^{(2)}\right)\right)$ for the infinitely repeated game happens when

$$
\begin{aligned}
x_{2}^{(2)} g_{1}-x_{1}^{(2)} c_{1} K_{1} & =\delta_{1} x_{2}^{(1)} g_{1}-x_{1}^{(1)} c_{1} K_{1} \\
x_{1}^{(1)} g_{2}-x_{2}^{(1)} c_{2} K_{2} & =\delta_{2} x_{1}^{(2)} g_{2}-a_{2}^{(2)} c_{2} K_{2}, \\
\text { where } & x_{1} \frac{K_{1}}{P_{1}}+x_{2} \frac{K_{2}}{P_{2}}=\tau .
\end{aligned}
$$

Since the two users take turn to make the first offer, the time-restricted bargaining strategy $\left(x_{1}^{*}, x_{2}^{*}\right)$ is

$$
\begin{aligned}
& x_{1}=\frac{1+m}{2} \times \frac{\left(1-\delta_{1}\right) \frac{P_{2}}{K_{2}} g_{1} \tau}{(m-1) K_{1} c_{1}+\left(m-\delta_{1}\right) \frac{K_{1} P_{2}}{K_{2} P_{1}} g_{1}} \\
& x_{2}=P_{2} \frac{\tau-x_{1} \frac{K_{1}}{P_{1}}}{K_{2}}, \text { where } m=\frac{g_{2}+c_{2} K_{2} \frac{P_{2}}{P_{1}}}{\delta_{2} g_{2}+c_{2} K_{2} \frac{P_{2}}{P_{1}}} .
\end{aligned}
$$

It is clear that the bargaining solution in (8) depends on the knowledge of both users' types, i.e., the private information, which is unavailable. Both players know the discount factors $\delta_{1}, \delta_{2}$ since the discount factors only depend on the chunks to be requested, which is the information the two users have to exchange. Although at the beginning, the users do not know each other's type, they can probe it during the bargaining process using the following mechanism: Let $T_{1}$ be $u_{1}$ 's type, which is only known by $u_{1}$, let $T_{2}$ be $u_{2}$ 's type and $T(j)$ is the $j^{\text {th }}$ type. At the first bargaining stage, without loss of generality, let $u_{1}$ be the first mover. $u_{1}$ calculates all the bargaining equilibriums $\left(a_{1}^{(1)}\left(T_{1}, T(j)\right), a_{2}^{(1)}\left(T_{1}, T(j)\right)\right)$ for $j=1,2,3$ corresponding to the three possible types of $u_{2}$. Then $u_{1}$ chooses the the equilibrium $j^{\prime}$ that gives highest $p_{j^{\prime}} \pi_{1}\left(a_{1}^{(1)}\left(T_{1}, T\left(j^{\prime}\right)\right), a_{2}^{(1)}\left(T_{1}, T\left(j^{\prime}\right)\right)\right)$. $u_{2}$ will accept the offer if $\pi_{2}\left(a_{1}^{(1)}\left(T_{1}, T(j)\right), a_{2}^{(1)}\left(T_{1}, T(j)\right)\right)$ is larger than or equal to $\pi_{2}\left(a_{1}^{(1)}\left(T_{1}, T_{2}\right), a_{2}^{(1)}\left(T_{1}, T_{2}\right)\right)$. If not, $u_{2}$ will offer back $\left(a_{1}^{(2)}\left(T_{1}, T_{2}\right), a_{2}^{(2)}\left(T_{1}, T_{2}\right)\right)$ and reach the agreement. Thus after the first bargaining stage in the first chunk-requesting round, $u_{2}$ knows $u_{1}$ 's type, and since $u_{2}$ will make the first move in next round, after 2 rounds, the both users have the information of each other's type.

Cheat On Private Information: Since users know each other's private information $\left(g_{i}, c_{i}\right)$ by the offers they made, users can cheat by making different offer. First, let we exam whether the time-restricted bargaining solution in (8) is cheat-proof with respect to $\left(g_{i}, c_{i}\right): \pi_{2}$ 
increases when $x_{2}$ decreases, which can be achieved by increasing $x_{1}$ or decreasing $P_{2} . \partial x_{1} / \partial m$ is always less than 0 , and $m$ is a monotonely increasing function of $g_{2}$ and is a monotonely decreasing function of $c_{2}$. Thus $u_{2}$ can have higher payoff by making the bargain offer using lower $g_{2}$, higher $c_{2}$, and lower $P_{2}$. Similarly, $u_{1}$ can also achieve higher utility by offering the equilibrium based on lower $g_{1}$, higher $c_{1}$, and lower $P_{1}$.

As the consequence that both players cheat with respect to $c_{i}$ and $g_{i}$, from the above analysis, both players will bargain based on the minimum value of $g_{i}$ and maximum value of $c_{i}$. Since we have assumed that $g_{i} \geq c_{i} K_{i}$, and $P_{i} \geq P_{\min }$, both players will make the offer based on $g_{i}=c_{i} K_{i}=C_{\max }$, and $P_{i}=P_{\min }$, thus the solution (8) becomes:

$$
\begin{aligned}
& x_{1}^{*}=\frac{\left(\delta_{2}+3\right)\left(1-\delta_{1}\right)}{2\left(4-\left(1+\delta_{1}\right)\left(1+\delta_{2}\right)\right)} \times \frac{\tau}{M / B \log \left(1+P_{\min } / \sigma_{n}^{2}\right)}, \\
& x_{2}^{*}=\frac{x_{1}^{*}}{M / B \log \left(1+P_{\min } / \sigma_{n}^{2}\right)}-
\end{aligned}
$$

which implies that both players should always cooperate with each other. It is clear that solution in (9) forms an Nash Equilibrium, is Pareto-Optimal, and is cheat-proof with respect to private information $g_{i}$ and $c_{i}$. Note that the user whose discount factor is closer to 1 has advantage, and if $\delta_{1}=\delta_{2}$, then $x_{1}^{*}=x_{2}^{*}=$ half number of chunks can be transmitted in $\tau$ seconds.

Cheat On Buffer Information: The other way of cheating is to cheat on buffer information, that is, although player i has chunk $k$ in the buffer, he/she does not report it to its opponent, so that reduce the number of requests from its opponent. However, hiding the chunk that the other user needs to prevent he/her from asking will increase the other user's discount factor based on (6), thus the cheating user will be in disadvantage in bargaining. Therefore, the users have no incentives to cheat on buffer information.

Based on the above analysis, we can conclude that, in the twoplayer wireless live-streaming game, in order to maximize each user's own payoff and be resistant to possible cheating behavior, for each player in each round, it should always agree to send the requested chunks up to the bargained chunk-requesting quota as in (8). We refer to the above strategy as two-player cheat-proof wireless live streaming cooperation strategy.

\section{SIMULATION RESULTS}

In our simulations, we use a Wi-Fi network as an example. The link from the wireless router to the Internet is a DSL link with $1.5 \mathrm{Mbits}$ download bandwidth. There are total 10 users in the network using live-streaming service without cooperation, and another 5 users using Internet resources at the same time. We fix the ration between laptop, PDA, and cellphone users as 1:2:2. The video is initially stored at an original server with an upload bandwidth of $3 \mathrm{Mbps}$, and there are other 400 users in the Internet watching the same live stream. The request round is 1 second and the buffer length is $10 \mathrm{sec}-$ onds. We choose the "Foreman" video sequence $(352 \times 288)$ resolution with frame rate $60 \mathrm{frame} / \mathrm{sec}$. We encode the video into a single layer bitstream with $150 \mathrm{kbps}$, and divide the video into chunks of 0.1 second, thus the chunk size is $\mathrm{M}=15 \mathrm{kbits}$. Among those 10 livestreaming users in the Wi-Fi network, we randomly choose two users to cooperate using the two-player cheat-proof wireless live streaming cooperation strategy. We run the simulation 40 times with 40 different pairs of users. We set $g_{i}=1=C_{\max }=0.8 c_{\text {cellphone }} * K_{i}$, $c_{\text {cellphone }}: c_{P D A}: c_{\text {laptop }}=1: 0.9: 0.4, P_{\text {min }}=100 \mathrm{~mW}$, noise power $=20 \mathrm{~mW}$, and bandwidth $\mathrm{B}=100 \mathrm{kHz}$. Discount measure in (6) is set to be 0.1 .

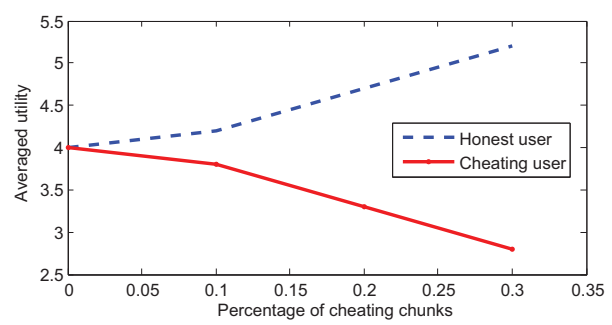

Fig. 3. Utilities of cheating player and honesty player versus number of cheating chunks in buffer

Figure 4 shows the averaged utilities of the player cheating on buffer information versus number of cheated chunks in the buffer while the other player is always honest. The utility is averaged over all the simulation runs and all the rounds. It is clear that the more chunks the player cheats on, the lower its own payoff, thus the best policy is being honest, which shows the cheat-proof property of our proposed cooperation strategy. And also, from the simulation, the averaged number of chunks per second of peers without cooperation is 3.7 , and that of the two peers with cooperation is 6.2 , which is much higher than peers without cooperation. Thus, cooperation can help users in wireless live-streaming social networks significantly improve the quality of their received video sequences.

\section{CONCLUSION}

In this paper, we investigate cooperation stimulation in wireless livestreaming social networks under a game theoretic framework. An illustrating two-player Bayesian game is studied, and different optimality criteria, including Pareto-Optimal and time-restricted bargaining solution is performed to refine the obtained equilibriums. And finally, a cheat-proof cooperation strategy is derived which provide the users in wireless live streaming social network an secured inventive to cooperate.

\section{REFERENCES}

[1] "The software is available at http://www.pplive.com/en/index.html," .

[2] G. Owen, Game Theory, Academic Press, 3rd edition, 2007.

[3] O.Karonen and J.K.Nurminen, "Cooperation incentives and enablers for wireless peers in heterogeneous networks," IEEE International Conference on Communications, , no. 134-138, May 2008.

[4] T. Ozbilgin and M.O. Sunay, "On the capacity of wireless peer-to-peer networks with transmitter and receiver cooperation," IEEE International Symposium on Personal, Indoor and Mobile Radio Communications, , no. 1-5, Sept 2007.

[5] Chiranjeeb Buragohain, Divyakant Agrawal, and Subhash Suri, "A game theoretic framework for incentives in $\mathrm{p} 2 \mathrm{p}$ systems," In Proceeding of the International Conference on Peer-to-Peer Computing, pp. 48-56, Sep 2003.

[6] S. Jun and M. Ahamad, "Incentives in bittorrent induce free riding," In Proceeding of the 2005 ACM SIGCOMM workshop on Economics of peer-to-peer systems, 2005.

[7] D. Qiu and R. Srikant, "Modeling and performance analysis of bittorrent-like peer-to-peer networks," In Proceedings of SIGCOMM 2004, 2004.

[8] Zhengye Liu, Yanming Shen, Shivendra Panwar, Keith Ross, and Yao Wang, "Using layered video to provide incentives in $\mathrm{p} 2 \mathrm{p}$ live streaming," ACM Special Interest Group on Data Communication, August 2007.

[9] Ahsan Habib and John Chuang, "Incentive mechanism for peer-topeer media streaming," International Workshop on Quality of Service (IWQoS), pp. 171-180, June 2004.

[10] M.J. Osborne and A. Rubinste, A Course in Game Theory, The MIT Press, 1994. 